\title{
The influence of films thickness on hydrogenation behavior of titanium thin films
}

\author{
Ervin Tal-Gutelmacher • Astrid Pundt • \\ Reiner Kirchheim
}

Received: 31 December 2009/Accepted: 15 July 2010/Published online: 24 July 2010

(C) The Author(s) 2010. This article is published with open access at Springerlink.com

\begin{abstract}
Titanium films of different thicknesses were prepared on sapphire substrates in an UHV chamber, by means of ion beam sputter deposition at room temperature, under Ar-atmosphere at the pressure of $1.5 \cdot 10 \mathrm{E}^{-4} \mathrm{mbar}$. For electrochemical hydrogen loading, the films were covered by a $30-\mathrm{nm}$ thick layer of Pd in order to prevent oxidation and facilitate hydrogen absorption. In situ stress measurements were conducted during step-by-step electrochemical hydrogen charging of the films. XRD measurements using a Phillips X-Pert diffractometer with a Co-K $\alpha$ radiation were performed before and after hydrogenation in order to investigate the effect of hydrogen loading on the microstructure. The phase boundaries, as well as the stress and strain development during hydrogen absorption, depend strongly on the thickness of the films. The main characteristics of absorption behavior of hydrogen, as well as the thermodynamics and phase boundaries of titanium-hydrogen thin films are discussed in detail with specific emphasis on the influence of films thickness. The obtained results are also compared to literature data on the widely studied titanium-hydrogen bulk system. Shifted phase boundaries and narrowed two-phase field appear in $\mathrm{Ti}-\mathrm{H}$ film system, which are mainly attributed to the microstructural contribution, as well as to the large stresses in the GPa-range that built up between the films and their substrate.
\end{abstract}

\section{Introduction}

Based on the high affinity for of Ti for hydrogen and its isotopes, the properties of $\mathrm{Ti}-\mathrm{H}$ bulk system have been

E. Tal-Gutelmacher $(\bowtie) \cdot$ A. Pundt $\cdot$ R. Kirchheim Institute for Materials Physics, Goettingen 37077, Germany

e-mail: ervin@ump.gwdg.de widely studied and reviewed [1-5]. However, to our knowledge, this is not the case with titanium thin films, despite both the fundamental and applicative interest that this system manifests. For example, thin films of titanium hydride (deuteride) are considered as potential candidates for hydrogen storage in catalytic or energetic reactions [6]. Recently, titanium hydride layers grown epitaxially on the surface of titanium implants, which were found recently to be a promising candidate for biomedical applications since the plastically deformed hydride layer was able to accommodate externally applied stresses without disturbing the titanium body structure [7].

From the fundamental point of view, by reducing the system size to the nanometer-scale, the surface-to-volume atom ratio, and for nanocrystalline materials, the interface-to-volume atom ratio, becomes large. Therefore, surface and interface contributions become more relevant and are expected to change the system properties compared to bulk. Thin films are usually deposited onto substrates that are either single crystals, polycrystals, or amorphous. The deposition conditions, e.g., technique, temperature, pressure, kinetic energy of the atoms, and the lattice misfit between film and substrate affect the films microstructure and the intrinsic stress of the asgrown thin film. The microstructure of thin films can be rather different and contain numerous microstructural defects. Films' grains can be of all sizes, ranging between that of nanocrystals with a high density of grain boundaries, to single crystals for epitaxial films. The mean grain size can also change in the vertical direction. Even when the grain size is maximized by epitaxial film growth, dislocations are generated during the film's growth process to adjust the lattice mismatch between the film and the substrate. In the vicinity of these defects, the hydrogen solubility is altered compared to conventional interstitial 
lattice sites [8, 9]. Furthermore, the interpretation of results in metal-hydrogen nanosized systems is complicated because the exact determination of the hydrogen concentration is difficult [10]. However, using electrochemical hydrogen charging technique under the proper conditions, the hydrogen concentration can be accurately determined via Faraday's law [9-11].

In this study, the thermodynamics of ion beam sputtered titanium films of different thicknesses during hydrogen absorption are investigated by electromotive force (EMF) measurements and in situ stress measurements.

\section{Experimental procedure}

Titanium films of 100 and $220 \mathrm{~nm}$ thickness were prepared on 0.2-mm thick and 27-mm long (0001) oriented sapphire $\left(\mathrm{Al}_{2} \mathrm{O}_{3}\right)$ substrates, in an UHV chamber by ion beam sputter deposition, at room temperature. The base pressure in the chamber was $10^{-8}$ mbar, whereas the deposition took place under Argon atmosphere at the pressure of $1.5 \cdot 10^{-4}$ mbar. To meet the geometry restrictions for stresses evaluation during hydrogen absorption, the dimensions of the as-sputtered films were $25 \times 0.5 \mathrm{~mm}$. In order to prevent oxidation and facilitate the electrochemical hydrogen charging, Ti-films were covered with a 30-nm thick Pd cap layer, which was also deposited at room temperature, under the same aforementioned conditions. The film's thickness was measured by profilometry. The crystal structure of the assputtered films, as well as after hydrogenation, was investigated by X-ray diffraction using a Phillips X-Pert diffractometer with a $\mathrm{Co}-\mathrm{K} \alpha$ radiation. In order to release the residual hydrogen that might have been picked up during the deposition process, prior to electrochemical hydrogenation, the Ti-films were discharged following the procedure described elsewhere [12]. Then, step-by-step hydrogen charging [10] was performed electrochemically in a 1:2 (vol.) $\mathrm{H}_{3} \mathrm{PO}_{4}$ :Glycerine electrolyte, at room temperature. An $\mathrm{Ag} / \mathrm{AgCl}$ (sat.) and Pt wires were used as the reference and the counter electrode. The current density was $0.05 \mathrm{~mA}$ during each loading step. The electrolyte was carefully cleaned from oxygen by prior Argon gas bubbling, so that no hydrogen losses due to $\mathrm{H}_{2} \mathrm{O}$ formation occurred. The hydrogen concentration in the films was calculated from the measured electric charge by using Faraday's law. Neither buckling, nor detachment of the film from the substrate, was observed after hydrogen loading, indicating good adhesion between the Ti-films and the sapphire substrates. The stresses developing in the film during hydrogen loading were in situ determined by measuring the deflection of the substrate using the setup described elsewhere [13], calculating its radius of curvature and inserting it to Stoney's equation [14]:
$\sigma=\frac{-E_{\mathrm{s}}}{6\left(1-v_{\mathrm{s}}\right)} \cdot \frac{t_{\mathrm{s}}^{2}}{t_{\mathrm{f}} R}$

where $E_{\mathrm{s}}$ and $v_{\mathrm{s}}$ are the Young's Modulus and the Poisson's ratio of the substrate, $t_{\mathrm{s}}$ and $t_{\mathrm{f}}$ are, respectively, the thicknesses of the substrate and the film, and $R$ is the substrate's radius of curvature. Formula (1) follows from an analysis of a film-substrate system, which is based on several assumptions [15, 16]. The main assumptions are (i) both the film thickness and substrate thicknesses are small compared to the lateral dimensions; (ii) the film thickness is much less than the substrate thickness; (iii) the substrate material is homogeneous, isotropic, and linearly elastic, and the film material is isotropic; (iv) edge effects near the periphery of the substrate are inconsequential; (v) all physical quantities such as Young's modulus and Poisson's ratio are invariant under change in positions parallel to the interface; (vi) all stress gradients in the thickness direction vanish throughout the material; and (vii) the strains and the rotations are small. To meet the geometry demands, first, the lateral dimensions of both Ti-films and their sapphire substrates were 27 and $25 \mathrm{~mm}$, i.e., larger by five and three orders of magnitude, respectively, compared to the films and substrates thicknesses. Secondly, the films thickness is smaller by three orders of magnitude than the substrates thickness.

\section{Results and discussion}

Besides the reflections of the sapphire substrate and the Pdcoating layer which are observed in each graph, in the XRD patterns of 220- and 100-nm Ti-films (Fig. 1), the only diffraction signal from the Ti-film is a broad peak around $43.3^{\circ}$, which in principle might be attributed to the (0002) hexagonal $\alpha$-Ti. From the Full Width at Half Maxima (FWHM) of the broad (0002) Ti-peak, the calculated out-of-plane grain size (diameter) using DebyeScherrer formula was found to be within 13-15 nm for both films.

Both films exhibit a preferred (0002) orientation, i.e., most grains are oriented so that the $\{0001\}$ planes are parallel with the substrate. This orientation is the fairly common growth orientation in evaporated and sputterdeposited hcp metals [17]. Ti-films sputtered at roomtemperature resulted in an out-of-plane $c$-textured structure, with a (0002) preferred growth orientation, though with a much reduced degree of in-plane orientation. In the work of Peddada et al. [18], $\alpha$-Ti thin films, deposited by e-beam physical vapor deposition at $723 \mathrm{~K}$, were found to grow epitaxially on sapphire substrates. The deposition temperature plays a significant role in the degree of epitaxy and higher temperature improves the crystal 


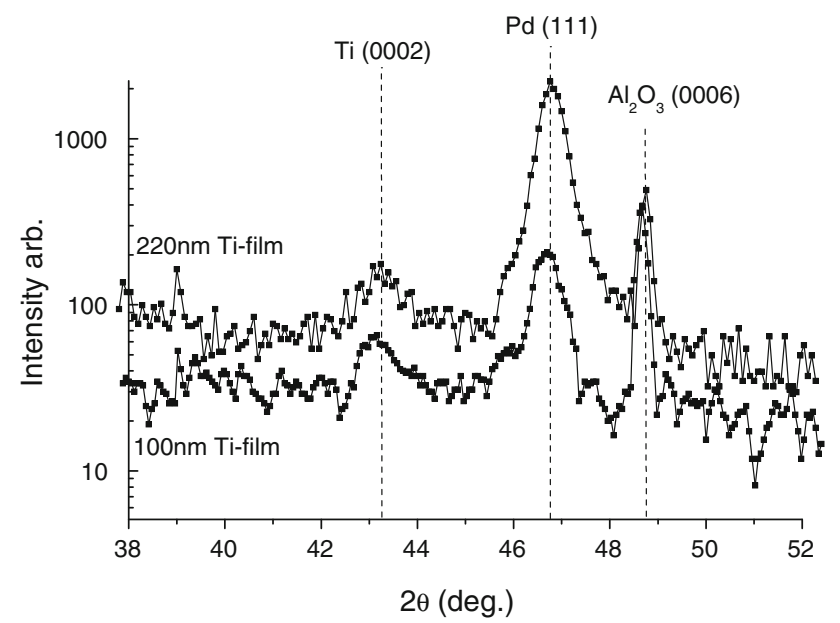

Fig. 1 XRD patterns of 100- and 220-nm thick Ti-films sputtered at $\mathrm{RT}$ on (0001) $\mathrm{Al}_{2} \mathrm{O}_{3}$ substrates

quality of the as-grown film. A similar observation was reported on epitaxial and textured Mg-thin films, deposited on sapphire and glass substrates [19, 20].

The results of EMF measurements during hydrogen charging of Ti-films, carried out at room temperature, are shown in Fig. 2. All the results shown below are those of the first charging. The phase boundaries, obtained from these plots, are summarized in Table 1.

Up to a hydrogen concentration of, respectively, 0.1 and $0.05 \mathrm{H} / \mathrm{Ti}$, the EMF-pressure-concentration curves of 220and 100-nm thick Ti-films reveal the presence of the $\alpha$ phase. When the hydrogen absorbed exceeds this concentration, the EMF/pressure values remain constant with the increase of hydrogen concentration, indicating the plateau, i.e., two-phase region. From theses curves, the plateau ranges from 0.1 to $0.4 \mathrm{H} / \mathrm{Ti}$ for the thicker, $220-\mathrm{nm}$ film, whereas for the $100-\mathrm{nm}$ Ti-film it ranges from 0.05 to $0.1 \mathrm{H} / \mathrm{Ti}$ (see Fig. 2b, c). Beyond these concentrations, hydride precipitation occurs, as revealed by the further increase of EMF/pressure values with hydrogen concentration. With further hydrogenation above $1.2 \mathrm{H} / \mathrm{Ti}$, bubbling was observed to occur on the samples surfaces due to the saturation of the Pd-capping layer. Therefore, the measured EMF/pressure values correspond to mainly the charging of the Pd-capping layer and not to the hydrogen absorbed within Ti-films. Since in these circumstances it is not possible to accurately evaluate the concentration of hydrogen within the films, this part of the curve should be disregarded.

The reduction of the Ti-H system size from bulk to thin films results in significant changes. In comparison to $\mathrm{Ti}-\mathrm{H}$ bulk system, Ti-H films exhibit significant differences. The plateau pressure of the samples is elevated compared to that of $\mathrm{Ti}-\mathrm{H}$ bulk system. As, to our knowledge, no experimental data at room temperature is available in the (a)
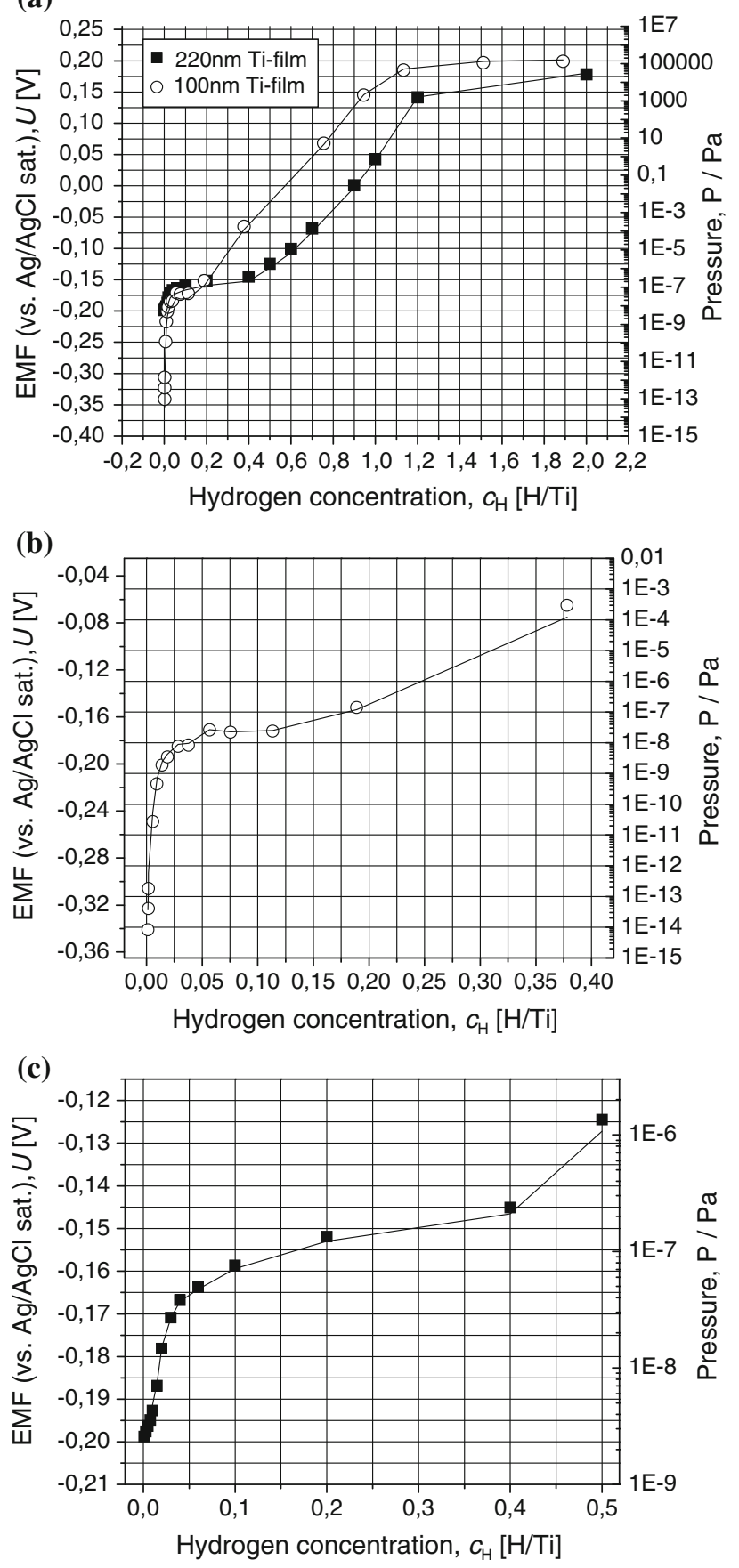

Fig. 2 a EMF-pressure-concentration curves of 100- and 220-nm thick Ti-films deposited on (0001) $\mathrm{Al}_{2} \mathrm{O}_{3}$ substrates. Enlargement of the $\alpha$-phase and the plateau region is shown in $\mathbf{b}$ for $100-\mathrm{nm}$ thick Tifilm and in c for 200-nm thick Ti-film

literature concerning bulk $\mathrm{Ti}-\mathrm{H}$ system, a plateau pressure of $10^{-11} \mathrm{~Pa}$ for bulk $\mathrm{Ti}$ at room temperature was extrapolated from reference [21]. The increased plateau pressure observed in the EMF-pressure-concentration curves of the films can be attributed to either stress contributions or to a metastable hydride phase $[9,10]$. The phase boundaries are 
Table 1 EMF measurements of hydrogen solubility at room temperature in Ti-films of different thicknesses

\begin{tabular}{llll}
\hline Sample & $\begin{array}{l}\alpha \text {-phase } \\
\text { limit } \\
{[\mathrm{H} / \mathrm{Ti}]}\end{array}$ & $\begin{array}{l}\text { Miscibility } \\
\text { gap [H/Ti] }\end{array}$ & $\begin{array}{l}\text { Hydride's } \\
\text { precipitation } \\
{[\mathrm{H} / \mathrm{Ti}]}\end{array}$ \\
\hline $\begin{array}{l}220-\mathrm{nm} \text { Ti-film on }(0001) \\
\mathrm{Al}_{2} \mathrm{O}_{3}\end{array}$ & 0.1 & $0.1-0.4$ & $>0.4$ \\
$\begin{array}{l}100-\mathrm{nm} \text { Ti-film on }(0001) \\
\mathrm{Al}_{2} \mathrm{O}_{3}\end{array}$ & 0.05 & $0.05-0.1$ & $>0.1$ \\
\begin{tabular}{l}
$\mathrm{Ti}-\mathrm{H}$ bulk system $[1,2]$ \\
\hline
\end{tabular} & $4 \times 10^{-4}$ & $4 \times 10^{-4}-1.5$ & 1.5 \\
\hline
\end{tabular}

Values for bulk Ti-H system are also shown for comparison

shifted, $\alpha$-phase limit is increased and the miscibility gap (two phase region) is narrowed.

A similar trend of the system's size effect is observed when comparing Ti-H film system of different thicknesses. The influence of film thickness on the thermodynamic isotherms is clearly observed; the hydride precipitation occurs at lower concentrations with the decrease in the films thickness, leading to a more narrowed miscibility gap in 100-nm thick Ti-film. The hydride formation in this film starts at lower hydrogen concentrations, and also the $\alpha$ phase limit is reduced in comparison to the 220-nm thick films. As expected, the plateau pressure does not change with the film thickness. Hydrogen solubility in the hydride phase is lower for the thinner film.

The results of in situ stress measurements and hydrogen charging, i.e., EMF measurements, are plotted in Fig. 3.

Large compressive stresses occur during hydrogen charging of Ti thin films. The maximum measured stress for each film is higher than the theoretical shear strength of bulk-Ti (generally estimated as $G / 30$ and hence, corresponding to a value of $1.4 \mathrm{GPa}$, where $G$ is the shear modulus of titanium equaling $44 \mathrm{GPa}$ ). The total hydrogen related stress for these Ti-films is -2.2 and $-1.8 \mathrm{GPa}$ for, respectively, 100- and 220-nm thick films, indicating that the overall stress is larger for the thinner film. The trends of the curves are approximately similar for both films in the $\alpha$ phase and the hydride region, while the distinctive difference is observed in the curves' slope at the two-phase region. In the $\alpha$-phase region (for low concentrations below $\mathrm{H} / \mathrm{Ti}=0.1$ ), the compressive stress increases linearly with hydrogen concentration in both plots. Based on the elastic linear theory [22, 23], since the films are clamped to elastically hard substrates, the elastic volume expansion due to hydrogen absorption can take place only in out-ofplane direction $z$ at low $\mathrm{H}$ concentrations. Thus, the linear increase of compressive stress was confirmed in our stressconcentration plots. At a concentration of 0.09 and $0.08 \mathrm{H} / \mathrm{Ti}$ for, respectively, 220- and 100-nm thick Ti-films, corresponding to the $\alpha$-phase limit, the slope of both curves changes. The deviation from elastic behavior
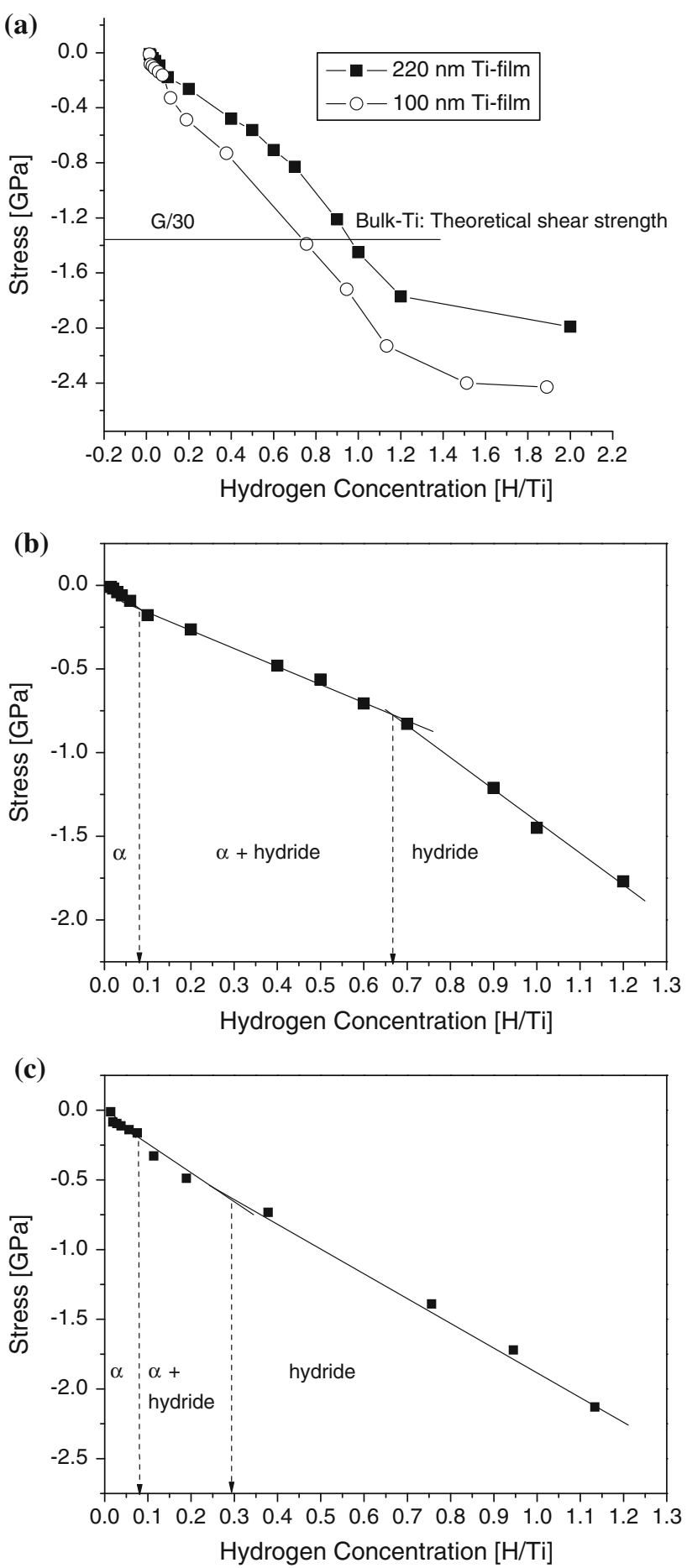

Fig. 3 a Stress-concentration curves of 220- and 100-nm thick Ti-films during hydrogen absorption, simultaneously obtained with EMF measurement. Slope variations occur at phase boundaries, as shown by the respective curves fitting in (b) and (c)

with the increase in hydrogen concentration can be explained by plastic deformation processes, which are attributed to the presence of misfit dislocations near the interface and to the emission of extrinsic dislocation loops 
Table 2 Phase boundaries of H-Ti-films of different thicknesses, as obtained from the change in the slope of stress-concentration curves

\begin{tabular}{llll}
\hline Sample & $\begin{array}{l}\alpha \text {-Phase } \\
\text { limit } \\
{[\mathrm{H} / \mathrm{Ti}]}\end{array}$ & $\begin{array}{l}\text { Miscibility } \\
\text { gap [H/Ti] }\end{array}$ & $\begin{array}{l}\text { Hydride's } \\
\text { precipitation } \\
{[\mathrm{H} / \mathrm{Ti}]}\end{array}$ \\
\hline $\begin{array}{l}220-\mathrm{nm} \text { Ti-film on }(0001) \\
\mathrm{Al}_{2} \mathrm{O}_{3}\end{array}$ & 0.09 & $0.09-0.66$ & $>0.66$ \\
$\begin{array}{l}100-\mathrm{nm} \text { Ti-film on }(0001) \\
\mathrm{Al}_{2} \mathrm{O}_{3}\end{array}$ & 0.08 & $0.08-0.3$ & $>0.3$ \\
$\mathrm{Ti}-\mathrm{H}$ bulk system $[1,2]$ & $4 \times 10^{-4}$ & $4 \times 10^{-4}-1.5$ & 1.5 \\
\hline
\end{tabular}

Values for bulk Ti-H system are also shown for comparison

during phase transformation/hydride precipitation [24]. As the hydrogen concentration exceeds the miscibility gap, the slope of the stress curves changes again. It can clearly be seen that the change in the slope occurs at lower concentrations for the thinner (at $0.3 \mathrm{H} / \mathrm{Ti}$, in comparison to $0.66 \mathrm{H} / \mathrm{Ti}$ for $220-\mathrm{nm}$ thick film), indicating again that the miscibility gap is narrowed. Both films reveal a similar slope in the hydride phase. The slope change indicating further slow increase of the compressive stress above $1.2 \mathrm{H} / \mathrm{Ti}$ could be related to the charging of the Pd-capping layer, similar to the reports on Y-films [25].

The phase boundaries observed by the slope changes in stress-concentration curves of both 100- and 220-nm thick Ti-films are summarized in Table 2.

They correspond by only a very rough approximation to those obtained from their EMF-pressure-concentration curves. The difference is more significant for the thinner, 100-nm Ti-film. In both films, the dissimilarity is more pronounced in the hydrogen concentration value for hydride precipitation, which is found to be larger based on the change of slope in the stress-concentration curves. It should be mentioned that the change in the slope of the stress-concentration curve will be observed only when incoherent hydride precipitation occurs [23-25], whereas the precipitation of coherent hydrides will not result in such a change. This could explain the higher terminal hydrogen solubility for hydride precipitation obtained in these measurements in comparison to EMF-pressure-concentration curves. XRD results reported elsewhere [26, 27] of measurements, which were conducted after each charging step in order to study the microstructural changes induced by hydrogen absorption, confirm the presence of a two-phase region at $0.4 \mathrm{H} / \mathrm{Ti}$ in $220-\mathrm{nm}$ thick Ti-films, while the spectra of the next charging step to $0.7 \mathrm{H} / \mathrm{Ti}$ revealed the sole hydride peak. Hence, hydride precipitation for this film occurs at the range of hydrogen concentrations higher than 0.4 and lower than $0.7 \mathrm{H} / \mathrm{Ti}$. Nevertheless, more precise, i.e., in situ microstructural investigations, need to be carried out in order to determine the exact boundary concentration.

\section{Conclusions}

In this study, the main characteristics of hydrogen's absorption behavior, the thermodynamics, and phase boundaries of titanium-hydrogen thin films were investigated and compared to titanium-hydrogen bulk system. Hydrogen behavior in Ti-thin films revealed significant changes in comparison to bulk material. Shifted phase boundaries and narrowed miscibility gaps appeared, as confirmed by simultaneously EMF and stress measurements. Large compressive stresses develop in Ti-films during hydrogen absorption. In low hydrogen concentration range, $\alpha$-phase region, the films behave linear elastically, whereas with further hydrogenation, plastic deformation processes occur. The changes in the thermodynamic isotherms of Ti-films vary between the films of different thickness, and are attributed mainly to stress contributions. The influence of the film thickness is manifested in the narrower miscibility gap and hydride precipitation at lower hydrogen concentrations, which are correlated to the higher stress level in the thinner films.

Acknowledgements This study was carried out under the financial support of Alexander von Humboldt foundation.

Open Access This article is distributed under the terms of the Creative Commons Attribution Noncommercial License which permits any noncommercial use, distribution, and reproduction in any medium, provided the original author(s) and source are credited.

\section{References}

1. Numakura H, Coiwa M (1984) Acta Metall 32:1799

2. Woo GC, Weatherly CE, Coleman CE, Gillbert RW (1985) Acta Metall 33:1897

3. Paton NE, Williams JC (1976) In: Thompson AW, Bernstein IM (eds) Effect of hydrogen on behaviour of materials. AIME, New York, NY, p 409

4. Nelson HG (1996) In: Thompson AW, Moody NR (eds) Hydrogen effects in metals. TMS, Warrendale, PA, p 699

5. Tal-Gutelmacher E, Eliezer D (2004) Mater Trans 45:1594

6. Kirchheim R, Fromm E, Wicke E (1988) Metal-hydrogen systems: fundamentals and applications. Oldenburg Verlag, Germany

7. Conforto E, Caillard D, Aronsson BO, Descouts P (2004) Philos Mag 1:631

8. Pundt A (2004) Adv Eng Mater 6:11

9. Pundt A, Kirchheim R (2006) Annu Rev Mater Res 36:555

10. Kirchheim R (1988) Prog Mater Sci 32:261

11. Zuchner H (1970) Naturforschung A 25:1490

12. Tal-Gutelmacher E, Pundt A, Kirchheim R (2010) Scr Mater 62:709

13. Pundt A, Nikitin E, Pekarski P, Kirchheim R (2004) Acta Mater 52:1579

14. Stoney GG (1909) Proc R Soc Lond Ser A 82:172

15. Freund LB, Floro JA, Chason E (1999) Appl Phys Lett 74:1987

16. Finot M, Blech IA, Suresh S, Fujimoto H (1997) J Appl Phys $81: 3457$

17. Barret CS, Massalski TB (1963) Structure of metals. McGrawHill, New York, p 540 
18. Peddada SR, Robertson IM, Birnbaum HK (1997) J Mater Res 12:1856

19. Kelekar R, Giffard J, Kelly ST, Klemens BM (2007) J Appl Phys 101:114311

20. Ozgit C, Akyildiz H, Ozturk T (2010) Thin Solid Films doi: 10.1016/j.tsf.2010.01.023

21. Fromm E, Gebhardt E (1976) Gase und Kohlenstoff in Metallen. Springer-Verlag, Berlin, Heidelberg

22. Yang QM, Schmitz G, Fähler S, Krebs HU, Kirchheim R (1996) Phys Rev B 54:9131

23. Laudahn U, Fähler S, Krebs HU, Pundt A, Bicker M, Hülsen Uv, Geyer U, Kirchheim R (1999) Appl Phys Lett 74:647
24. Laudahn U, Pundt A, Bicker M, Hulsen Uv, Geyer U, Wagner T, Kirchheim R (1999) J Alloys Compd 293-295:490

25. Dornheim M, Pundt A, Kirchheim R, Molen SJ, Kooji ES, Kerssemakers J, Griessen R, Harms H, Geyer U (2003) J Appl Phys 93:8958

26. Tal-Gutelmacher E, Gemma R, Pundt A, Kirchheim R (2009) In: Somerday B, Sofronis P, Jones R (eds) Effects of hydrogen on materials. ASM International, Materials Park, OH, p 548

27. Tal-Gutelmacher E, Gemma R, Pundt A, Kirchheim R (2010) Acta Mater 58:3042 